\title{
The Convergence and Order of the 2-point Improved Block Backward Differentiation Formula
}

\author{
Hamisu Musa \\ Department of Mathematics and Computer Science, Faculty of Natural and Applied Sciences, Umaru Musa \\ Yar'adua University, P.M.B. 2218, Katsina, Katsina State, Nigeria
}

Abstract: This paper studied the 2-point improved block backward differentiation formula for solving stiff initial value problems proposed by Musa et al (2013) and further established the necessary conditions for the convergence of the method. It is shown that the method is both zero stable and consistent. The order of the method is also derived.

Keywords: Improved block BDF, Zero stability, consistency conditions for block method, convergence, order.

\section{INTRODUCTION}

Consider a system of stiff initial value problem (IVP) of the form:

$$
y^{\prime}=f(x, y) \quad y\left(x_{0}\right)=\eta \quad \eta=\left[\eta_{1}, \eta_{2}, \cdots, \eta_{m}\right]
$$

Such problems are encountered in the modelling of equations related to electrical circuits, vibrations, kinetics, chemical reactions etc. The definition of stiff problems has not been precise due to the fact that stiffness occurs in several applications of different nature. According to a definition in [9], no universally accepted definition of stiffness exists. Brugnano et al [10] compiled various definitions of stiffness according to the applications of different nature in which they occur. The most common phenomena that describes stiffness is when the eigenvalues of the jacobian of the system (1) differ greatly in magnitude.

Considerable effort in dealing with stiffness has led to the development of many implicit numerical methods e.g. [14], [17], [16], [13], [8], [12], [2]. One of the most popular methods is the Backward Differentiation Formula (BDF) [1]. The development of the BDF has led to various methods, including ones that produced sequence of approximations simultaneously (block methods) [3], [18], [15], [11]. Examples of block methods based on the BDF include the block backward differentiation formula (BBDF) developed in [20], block extended backward differentiation formula (BEBDF) developed in [4], improved block backward differentiation formula (IBBDF) developed in [5]. Convergence of block methods for solving (1) has been studied in [19], [4]. The IBBDF method proves to be efficient and one of the recent block method for stiff problems. This paper therefore studied the IBBDF and investigates its convergence properties and order.

The 2-point IBBDF is derived by modifying the special case of the following 2-point BBDF

$$
\sum_{j=0}^{3} \alpha_{j, i} y_{n+j-1}=h \beta_{k, i} f_{n+k} \quad k=i=1,2
$$

A parameter $\rho=1 / 2$ and a non-zero coefficient $\beta_{k-1, i} \neq 0$ (where $\beta_{k-1, i}=\rho \beta_{k, i}$ ) are introduced in (2) to come up with the following IBBDF method:

$$
\sum_{j=0}^{3} \alpha_{j, i} y_{n+j-1}=h \beta_{k, i}\left(f_{n+k}-\rho f_{n+k-1}\right), \quad k=i=1,2 .
$$

By choosing $\rho=1 / 2$, the following 2-point block formula is obtained:

$$
\begin{aligned}
& y_{n+1}=\frac{5}{4} y_{n}-\frac{1}{4} y_{n+2}+h\left(\frac{1}{2} f_{n}+f_{n+1}\right) \\
& y_{n+2}=\frac{1}{8} y_{n-1}-\frac{1}{2} y_{n}+\frac{11}{8} y_{n+1}+\frac{h}{2}\left(\frac{1}{2} f_{n+1}+f_{n+2}\right)
\end{aligned}
$$

Details on the derivations, stability and performance of the method can be found in [5]. 
In the remaining part of the paper, we derive the order of the method and show the convergence of the method.

\section{ORDER OF THE METHOD}

To derive the order of the method, define the formulae (4) in general matrix form as:

$$
\sum_{j=0}^{1} C_{j}^{*} Y_{m+j-1}=h \sum_{j=0}^{1} D_{j}^{*} F_{m+j-1}
$$

where $C_{0}^{*}, C_{1}^{*}, D_{0}^{*}, D_{1}^{*}, Y_{m-1}, Y_{m}, F_{m-1}$ and $F_{m}$ are defined by

$$
\begin{array}{rlr}
C_{0}^{*}=\left(\begin{array}{cc}
0 & -\frac{5}{4} \\
-\frac{1}{8} & \frac{1}{2}
\end{array}\right), & C_{1}^{*}=\left(\begin{array}{cc}
1 & \frac{1}{4} \\
11 & \\
-\frac{1}{8} & 1
\end{array}\right), & Y_{m}=\left(\begin{array}{c}
y_{n+1} \\
y_{n+2}
\end{array}\right), \\
D_{1}^{*}=\left(\begin{array}{cc}
1 & 0 \\
\frac{1}{4} & \frac{1}{2}
\end{array}\right), & F_{m}=\left(\begin{array}{c}
f_{n+1} \\
f_{n+2}
\end{array}\right) .
\end{array}
$$

Equation (5) can be written as

$$
\left(\begin{array}{cc}
0 & -\frac{5}{4} \\
-\frac{1}{8} & \frac{1}{2}
\end{array}\right)\left(\begin{array}{c}
y_{n-1} \\
y_{n}
\end{array}\right)+\left(\begin{array}{cc}
1 & \frac{1}{4} \\
11 & 1
\end{array}\right)\left(\begin{array}{l}
y_{n+1} \\
y_{n+2}
\end{array}\right)=h\left(\begin{array}{ll}
0 & \frac{1}{2} \\
0 & 0
\end{array}\right)\left(\begin{array}{l}
f_{n-1} \\
f_{n}
\end{array}\right)+h\left(\begin{array}{ll}
1 & 0 \\
\frac{1}{4} & \frac{1}{2}
\end{array}\right)\left(\begin{array}{l}
f_{n+1} \\
f_{n+2}
\end{array}\right)
$$

Let $C_{0}^{*}, C_{1}^{*}, D_{0}^{*}$ and $D_{1}^{*}$ be block matrices defined by

$$
\begin{array}{ll}
C_{0}^{*}=\left(\begin{array}{ll}
C_{0} & C_{1}
\end{array}\right), & C_{1}^{*}=\left(\begin{array}{ll}
C_{2} & C_{3}
\end{array}\right), \quad D_{0}^{*}=\left(\begin{array}{ll}
D_{0} & \left.D_{1}\right), \quad \text { and }
\end{array} D_{1}^{*}=\left(\begin{array}{ll}
D_{2} & D_{3}
\end{array}\right)\right.
\end{array}
$$

where

$$
\begin{array}{lll}
C_{0}=\left(\begin{array}{c}
0 \\
-\frac{1}{8}
\end{array}\right), & C_{1}=\left(\begin{array}{c}
-\frac{5}{4} \\
\frac{1}{2}
\end{array}\right), & C_{2}=\left(\begin{array}{c}
1 \\
-\frac{11}{8}
\end{array}\right), \\
D_{0}=\left(\begin{array}{l}
0 \\
0
\end{array}\right), & D_{1}=\left(\begin{array}{c}
\frac{1}{2} \\
0
\end{array}\right), & D_{2}=\left(\begin{array}{l}
\frac{1}{4} \\
\frac{1}{4}
\end{array}\right),
\end{array}
$$

\section{Definition 1}

The order of the block method (5) and its associated linear operator given by

$$
L[y(x) ; h]=\sum_{j=0}^{k=3}\left[C_{j} y(x+j h)-h \sum_{j=0}^{k}\left[D_{j} y^{\prime}(x+j h)\right]\right]
$$

is a unique integer $\mathrm{p}$ such that $E_{q}=0, q=0(1) p$ and $E_{p+1} \neq 0$; where the $E_{q}$ are constant (column) matrices defined by: 


$$
\begin{aligned}
& E_{0}=C_{0}+C_{1}+\cdots C_{k} \\
& E_{1}=C_{1}+2 C_{2}+k C_{k}-\left(D_{0}+D_{1}+\cdots+D_{k}\right) \\
& E q=\frac{1}{q !}\left(C_{1}+2^{q} C_{2}+k^{q} C_{k}\right)-\frac{1}{(q-1) !}\left(D_{1}+2^{q-1} D_{2}+k^{q-1} D_{k}\right)
\end{aligned}
$$

$q=2,3,4$

For $\mathrm{q}=0(1) 4$

$$
\begin{aligned}
& E_{0}=C_{0}+C_{1}+C_{2}+C_{3}=0 \\
& E_{1}=C_{1}+2 C_{2}+3 C_{3}-\left(D_{0}+D_{1}+D_{2}+D_{3}\right)=0 \\
& E_{2}=\frac{1}{2 !}\left(C_{1}+2^{2} C_{2}+3^{2} C_{3}\right)-\frac{1}{1 !}\left(D_{1}+2 D_{2}+3 D_{3}\right)=0 \\
& E_{3}=\frac{1}{3 !}\left(C_{1}+2^{3} C_{2}+3^{3} C_{3}\right)-\frac{1}{2 !}\left(D_{1}+2^{2} D_{2}+3^{2} D_{3}\right)=0 \\
& E_{4}=\frac{1}{4 !}\left(C_{1}+2^{4} C_{2}+3^{4} C_{3}\right)-\frac{1}{3 !}\left(D_{1}+2^{3} D_{2}+3^{3} D_{3}\right)=\left(\begin{array}{r}
\frac{1}{24} \\
-\frac{5}{48}
\end{array}\right) \neq\left(\begin{array}{l}
0 \\
0
\end{array}\right)
\end{aligned}
$$

Therefore, the formula (4) is of order 3, with error constant

$$
\left(\begin{array}{c}
\frac{1}{24} \\
-\frac{5}{48}
\end{array}\right)
$$

\section{CONVERGENCE OF THE METHOD}

Convergence is an essential property that every acceptable linear multistep method (LMM) must possess. This section shows the convergence of the method (4). According to [6], consistency and zero stability are the necessary conditions for the convergence of any numerical method. Lambert [7] also explained that consistency controls the magnitude of the local truncation error while zero stability controls the manner in which the error is propagated at each step of the calculation. A method which is not both consistent and zero stable is rejected outright and has no practical interest. We begin by showing that the method (4) is consistent. We start by presenting the following definitions and theorems related to the convergence of LMM.

Definition 2 (LMM)

A general linear multistep method (LMM) has the form:

$$
\sum_{j=0}^{k} \alpha_{j} y_{n+j}=h \sum_{j=0}^{k} \beta_{j} f_{n+j}
$$

where $\alpha_{j}$ and $\beta_{j}$ are constants and $\alpha_{k} \neq 0 . \alpha_{0}$ and $\beta_{0}$ cannot both be zero at the same time. For any $\mathrm{k}$ step method, $\alpha_{k}$ is normalised to 1 .

The method (10) is said to be explicit if $\beta_{k}=0$ and implicit if $\beta_{k} \neq 0$.

\section{Definition 3}

The first and the second characteristic polynomial of the LMM (10) are defined by: 


$$
\begin{aligned}
& \rho(\xi)=\sum_{j=0}^{k} \alpha_{j} \xi^{j} \\
& \sigma(\xi)=\sum_{j=0}^{k} \beta_{j} \xi^{j}
\end{aligned}
$$

respectively.

\section{Definition 4}

The linear difference operator L associated with the LMM (10) is defined by

$$
L[y(x) ; h]=\sum_{j=0}^{k}\left[\alpha_{j} y(x+j h)-h \beta_{j} y^{\prime}(x+j h)\right]
$$

where $y(x)$ is an arbitrary test function and it is continuously differentiable on $[a, b]$.

Expanding $y(x+j h)$ and $y^{\prime}(x+j h)$ as Taylor series about $\mathrm{x}$, and collecting common terms yields

$$
L[y(x) ; h]=C_{0} y\left(x_{n}\right)+C_{1} h y^{\prime}\left(x_{n}\right)+\cdots C_{q} h^{q} y^{(q)}\left(x_{n}\right)+\cdots
$$

Where the $C_{q}$ are constants given by

$$
\begin{aligned}
& C_{0}=\alpha_{0}+\alpha_{1}+\alpha_{2}+\cdots \alpha_{k} \\
& C_{1}=\alpha_{1}+2 \alpha_{2}+\cdots+k \alpha_{k}-\left(\beta_{0}+\beta_{1}+\beta_{2}+\cdots+\beta_{k}\right) \\
& \vdots \\
& C_{q}=\frac{1}{q !}\left(\alpha_{1}+2^{q} \alpha_{2}+\cdots+k^{q} \alpha_{k}\right)-\frac{1}{(q-1) !}\left(\beta_{1}+2^{q-1} \beta_{2}+\cdots+k^{q-1} \beta_{k}\right) \\
& \quad-\quad q=2,3, \cdots
\end{aligned}
$$

\section{Definition 5 (Consistency)}

The LMM (10) is said to be consistent if its order $p \geq 1$

It also follows from (14) that the LMM (10) is consistent if and only if

$$
\begin{aligned}
& \sum_{j=0}^{k} \alpha_{j}=0 \\
& \sum_{j=0}^{k} j \alpha_{j}=\sum_{j=0}^{k} \beta_{j}
\end{aligned}
$$

It also follows from (15) that the LMM (10) is consistent if and only if

$$
\begin{aligned}
& \rho(1)=0 \\
& \rho^{\prime}(1)=\sigma(1)
\end{aligned}
$$

\section{Definition 6}

The characteristic polynomial of the method (10) is defined as

$$
\pi(r, \bar{h})=\rho(\xi)-\bar{h} \sigma(\xi)=0
$$

Where $\bar{h}=\lambda h$ and $\lambda=\frac{\delta f}{\delta y}$.

\section{Definition 7 (Zero Stability)}

The LMM (10) is said to be zero stable if no root of the first characteristic polynomial $\rho(\xi)$ (defined by (11)) has modulus greater than one, and that every root with modulus one is simple. 
Theorem 1 (Convergence of LMM)

Henrici [6] gave the following theorems on convergence of LMM

(1) A necessary condition for convergence of the LMM (10) is that the modulus of no root of the associated polynomial $\rho(\xi)$ (given in (11)) exceeds 1 , and that the roots of modulus 1 be simple.

The condition thus imposed on $\rho(\xi)$ is called the condition of zero stability.

(2) A necessary condition for convergence of the LMM defined by (10) is that the order of the associated difference operator be at least 1 .

The condition that the order $p \geq 1$ is called the condition of consistency.

\subsection{Consistency of the method}

In this subsection, it is shown that the 2-point IBBDF satisfies the consistency conditions given in definition 5.

From what followed in section 2, it can be concluded that the order of the 2-point IBBDF method is greater than 1.

It now remains to show that the method is consistent.

The method (4) is consistent if and only if the following conditions are satisfied:

$$
\begin{aligned}
& \sum_{j=0}^{3} C_{j}=0 \\
& \sum_{j=0}^{3} j C_{j}=\sum_{j=0}^{3} D_{j}
\end{aligned}
$$

where the $C_{j^{\prime} s}$ and $D_{j^{\prime} s}$ are as previously defined.

Equation (18) then becomes

$$
\begin{aligned}
\sum_{j=0}^{3} C_{j} & =C_{0}+C_{1}+C_{2}+C_{3} \\
& =\left(\begin{array}{c}
0 \\
-\frac{1}{8}
\end{array}\right)+\left(\begin{array}{c}
-\frac{5}{4} \\
\frac{1}{2}
\end{array}\right)+\left(\begin{array}{c}
1 \\
-\frac{11}{8}
\end{array}\right)+\left(\begin{array}{l}
\frac{1}{4} \\
1
\end{array}\right) \\
& =\left(\begin{array}{l}
0 \\
0
\end{array}\right)
\end{aligned}
$$

Hence the first condition in (18) is satisfied.

$$
\begin{aligned}
\sum_{j=0}^{3} j C_{j} & =0 \times C_{0}+1 \times C_{1}+2 \times C_{2}+3 \times C_{3} \\
& =0 \times\left(\begin{array}{c}
0 \\
-\frac{1}{8}
\end{array}\right)+1 \times\left(\begin{array}{c}
-\frac{5}{4} \\
\frac{1}{2}
\end{array}\right)+2 \times\left(\begin{array}{c}
1 \\
-\frac{11}{8}
\end{array}\right)+3 \times\left(\begin{array}{c}
\frac{1}{4} \\
1
\end{array}\right) \\
& =\left(\begin{array}{l}
\frac{3}{2} \\
\frac{3}{4}
\end{array}\right)
\end{aligned}
$$




$$
\begin{aligned}
& \sum_{j=0}^{3} D_{j}=D_{1}+D_{2}+D_{3} \\
& =\left(\begin{array}{l}
0 \\
0
\end{array}\right)+\left(\begin{array}{l}
\frac{1}{2} \\
0
\end{array}\right)+\left(\begin{array}{c}
1 \\
\frac{1}{4}
\end{array}\right)+\left(\begin{array}{c}
0 \\
\frac{1}{2}
\end{array}\right) \\
& =\left(\begin{array}{l}
\frac{3}{2} \\
\frac{3}{4}
\end{array}\right) \\
& \text { Hence } \sum_{j=0}^{3} j C_{j}=\sum_{j=0}^{3} D_{j} \text {. }
\end{aligned}
$$

Thus, the second condition in (18) is also satisfied.

The consistency conditions are therefore met. Hence, the method is consistent. It now remains to show that the method is zero stable.

\subsection{Zero Stability}

The stability polynomial of the method (4) is given by:

$$
R(\bar{h}, t)=-\frac{5}{32}-\frac{1}{16} \bar{h}-\frac{19}{16} t-\frac{3}{2} \bar{h} t-\frac{1}{8} \bar{h}^{2} t+\frac{43}{32} t^{2}-\frac{23}{16} \bar{h} t^{2}+\frac{1}{2} \bar{h}^{2} t^{2}=0
$$

To derive the first characteristic polynomial, we set $\bar{h}=0$ in (22) to obtain:

$$
-\frac{5}{32}-\frac{19}{16} t+\frac{43}{32} t^{2}=0
$$

Solving (23) for t, we obtain the following roots:

$$
t=1, \quad t=-0.116279
$$

Thus according to definition 7 , the method (4) is zero stable.

Since the method (4) is both consistent and zero stable, it is thus convergent in accordance with Theorem 1.

\section{Conclusion}

The 2-point improved block backward differentiation formula is studied in this paper. The order of the method is shown to be 3 . The convergence of the method is also shown by proving that the method satisfied consistency conditions and that it is zero stable; thus validating its significance in solving stiff initial value problems.

\section{REFERENCES}

[1] C. Curtiss, J.O. Hirschfelder, Integration of stiff equations, Proceedings of the National Academy of Sciences of the United States of America. 38 (1952), pp. 235-243.

[2] D. Voss, S. Abbas, Block predictor-corrector schemes for the parallel solution of ODEs, Computers and Mathematics with Applications. 33 (1997)

$65-72$.

[3] G. Hall, J. M. Watt Modern Numerical Methods for Ordinary Differential Equations, Clarendon Press, 1976.

[4] H. Musa, M. B. Suleiman, F. Ismail, N. Senu, Z. B. Ibrahim The convergence and order of the 3 -point block extended backward differentiation formula, ARPN Journal of Engineering and Applied Sciences. 7(12) (2012) pp. 1539-1545.

[5] H. Musa, M. B. Suleiman, F. Ismail, N. Senu, Z. B. Ibrahim An improved 2 -point block backward differentiation formula for solving stiff initial value problems, AIP Conference Proceedings. 1522 (2013), pp. 211-220.

[6] Henrici, P. Discrete variable methods in ordinary differential equations, New York: Wiley, 11962.

[7] J. D. Lambert, Computational Methods in Ordinary Differential Equations, Chichester, New York, 1973.

[8] J. R. Cash, On the integration of stiff systems of ODEs using extended backward differentiation formulae, Numerische Mathematik. 34 (1980), 235-246.

[9] L. F. Shampine, S. Thompson, Stiff systems, Scholarpedia. 2(3) (2007), 28-55.

[10] L. Brugnano, F. Mazzia, D. Trigiante, Fifty years of stiffness, Recent Advances in Computational and Applied Mathematics. 2011, pp. $1-21$.

[11] L. Shampine, H. Watts, Block implicit one-step methods, Math. Comp. 23 (1969), pp. 731-740.

[12] G. D. Andria, G.D. Byrne, D.R. Hill, Natural spline block implicit methods, BIT Numerical Mathematics. 13 (1973), pp. $131-144$.

[13] G. Hall, M. Suleiman, A single code for the solution of stiff and nonstiff ODE's SIAM Journal on Scientific and Statistical 
Computing. 6 (1985), 684-694.

[14] J. Alvarez, J. Rojo, An improved class of generalized Runge-Kutta methods for stiff problems. Part I:The scalar case, Applied Mathematics and Computation. 130 (2002), pp. 537-560.

[15] S. O. Fatunla, Block methods for second order ODEs, International Journal of Computer Mathematics, 41 (1991), pp. 55-63.

[16] R. Ahmad, N. Yaacob, A.H.M. Murid, Explicit methods in solving stiff ordinary differential equations, International Journal of Computer Mathematics. 81 (2004), pp. 1407-1415.

[17] R. Alt, A-stable one-step methods with step-size control for stiff systems of ordinary differential equations, Journal of Computational and Applied Mathematics. 4 (1978), pp. 29-35.

[18] Z. A. Majid, M.B. Suleiman, Implementation of four-point fully implicit block method for solving ordinary differential equations, Applied Mathematics and Computation. 184 (2007), pp. 514-522.

[19] Z.B. Ibrahim, M. Suleiman, N.A.A.M. Nasir, K.I. Othman, Convergence of the 2-Point Block Backward Differentiation Formulas, Applied Mathematical Sciences. 5(70) (2011), pp. 3473-3480.

[20] Z.B. Ibrahim, K.I. Othman, M. Suleiman, Implicit r-point block backward differentiation formula for solving first-order stiff ODEs, Applied Mathematics and Computation. 186 (2007), pp. 558-565. 